> Les rétrovirus endogènes humains (human endogenous retrovirus - HERV) constituent une large part de notre génome dont ceux qui ont conservé un potentiel d'expression et restent «dormants » dans les conditions physiologiques. Ils peuvent être «réveillés » par des facteurs environnementaux activateurs de leur expression dont les mieux étudiés sont les infections causées par des microorganismes comme des virus de la famille des herpèsvirus. Cette activation peut ainsi conduire à l'expression de protéines pathogènes comme des protéines d'enveloppe appartenant aux familles HERV-W et HERV-K impliquées respectivement dans la sclérose en plaques (SEP) et la sclérose latérale amyotrophique (SLA). Des protéines rétrovirales endogènes peuvent aussi acquérir une fonction physiologique bénéfique pour l'homme. C'est le cas de la syncytine de la famille HERV-W qui est impliquée dans la formation du placenta. <

Les processus impliqués dans l'activation des rétrovirus endogènes humains (human endogenous retrovirus ou HERV) qui ont conservé un potentiel d'expression latent chez l'homme sont encore mal décrits [48] $(\rightarrow)$. En effet,

$(\rightarrow)$ Voir la Synthèse de J. Medina et $H$. Perron, $m / s n^{\circ} 2$, février 2017, page 151 bien que les quelques HERV avec des séquences codantes (cadres de lecture ouverts) soient épigénétiquement réprimés dans les conditions physiologiques, la littérature comporte plusieurs exemples d'agents infectieux capables d'activer leur expression comme des herpèsvirus, des lentivirus (comme le virus de l'immunodéficience humaine $[\mathrm{VIH}]$ ), des orthomyxovirus (tel que le virus de la grippe ou influenza) ou un parasite (comme Toxoplasma gondii) [1, 2].

Ces facteurs environnementaux peuvent abolir leur contrôle épigénétique et/ou « réveiller » leur expression. C'est ainsi que des publications scientifiques titrent : «l'ennemi de l'intérieur : des anciens rétrovirus

\title{
Des séquences rétrovirales endogènes dans le génome humain peuvent jouer un rôle physiologique ou pathologique
}

Julie Medina ${ }^{1}$, Benjamin Charvet ${ }^{2-7}$, Pascal Leblanc ${ }^{6,8}$ Raphaële Germi ${ }^{9}{ }^{10}$, Branka Horvat ${ }^{2-7}$, Patrice N. Marche ${ }^{11,12}$, Hervé Perron ${ }^{1,5,13}$

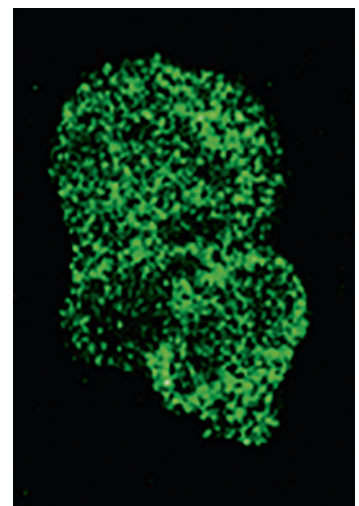

dormants (sont) réveillés» [3]. Nous illustrerons ici ce phénomène en prenant les Herpesviridae pour exemple.

\section{Induction de l'expression de rétrovirus endogènes par les Herpesviridae humains}

Bien que nos connaissances des mécanismes d'activation des séquences rétrovirales endogènes soient encore incomplètes, l'implication d'agents infectieux exogènes comme les Herpesviridae humains (que nous appellerons ici communément herpèsvirus) a été démontrée (Figure 1).

\section{Rôle des herpèsvirus sur la} transcription et l'expression de protéines ou virions endogènes Les herpèsvirus tels que HSV-I (herpes simplex virus-1), عBV (virus d'Epstein
${ }^{1}$ GeNeuro Innovation, Bioparc Laënnec, 60, avenue Rockefeller, 69008 Lyon, France ;

${ }^{2} \mathrm{CIRI}$, Centre International de Recherche en Infectiologie ; ${ }^{3}$ Inserm, Ull1l, Lyon, France ; ${ }^{4}$ CNRS, UMR5308, Lyon, France ; ${ }^{5}$ Université Lyon-1, Faculté de Médecine Laënnec, 69008 Lyon, France ;

${ }^{6}$ Institut NeuroMyogène (INMG), CNRS UMR5310, Inserm U1217, LBMC, École Normale Supérieure de Lyon, France ;

${ }^{7}$ Laboratoire d'Excellence ECOFECT, Lyon, France ;

${ }^{8}$ UMR 5239 CNRS-ENS, Université Lyon 1, Lyon, France ;

${ }^{9}$ Laboratoire de Virologie, Institut de Biologie et

Pathologie, CHU de Grenoble, France;

${ }^{10}$ Institut de Biologie Structurale, UMR 5075 CEA/ CNRS/UGA, Grenoble, France.

${ }^{11}$ Inserm U1209, Grenoble, France ${ }^{12}$ Institut Advance Biosciences, CNRS UMR5309, Université Grenoble Alpes

${ }^{13}$ GeNeuro, 18, chemin des Aulx, 1228 Plan-Les-Ouates, Genève, Suisse.

hperron@geneuro.com

julie.medina@geneuro.com hp@geneuro.com 


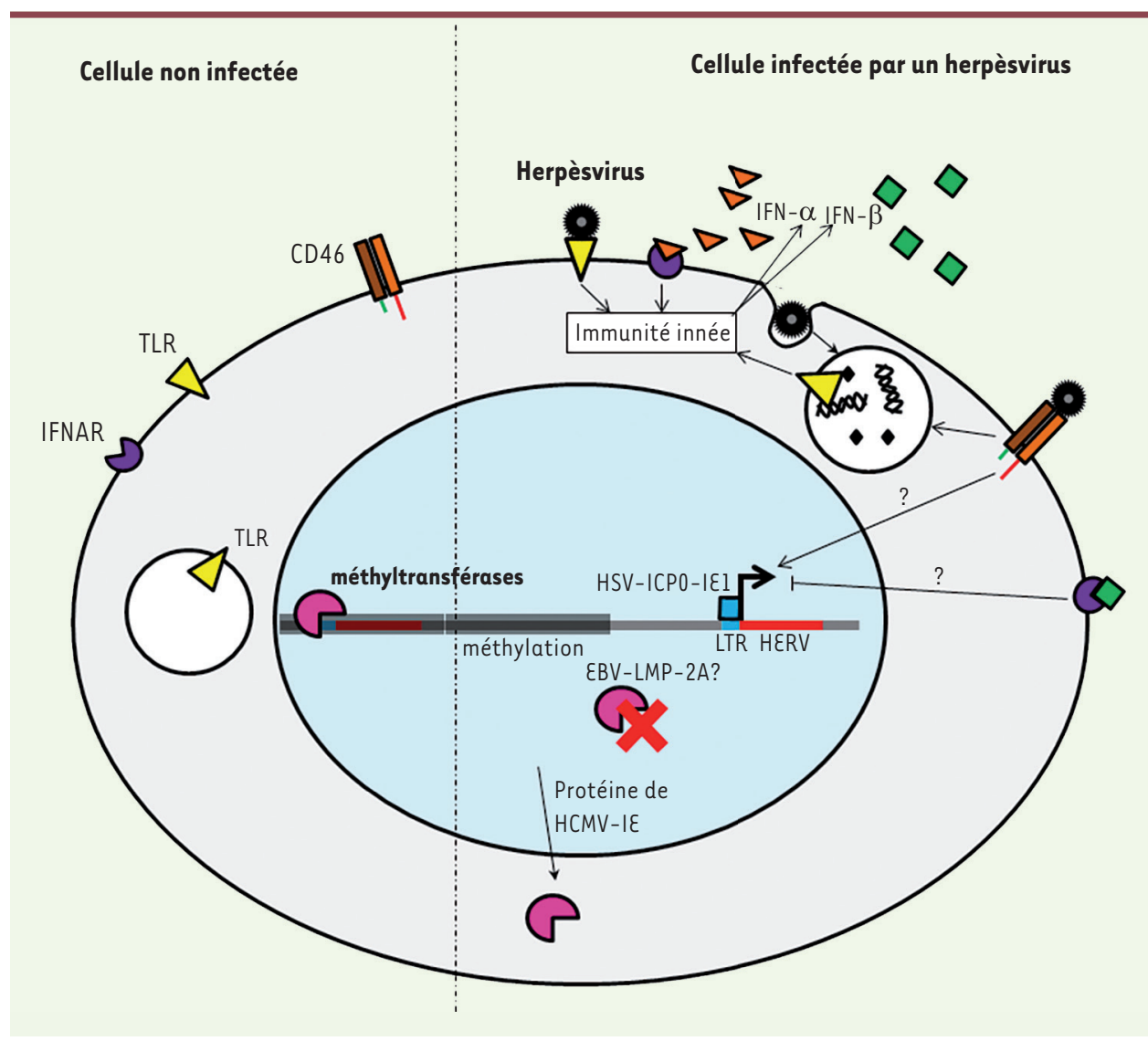

Figure 1. Représentation schématique des mécanismes de transactivation et de suppression du contrôle épigénétique dans une cellule infectée par les Herpesviridae (ou herpèsvirus). Les mécanismes connus pour les herpèsvirus $\varepsilon B V$ (virus d'Epstein-Barr), HSV-l (virus herpès simplex de type 1 ), HHV-6 (Herpesviridae humains 6) et HCMV (cytomégalovirus humain) sont illustrés. Comparaison des états cellulaires entre une cellule non infectée par les herpèsvirus (partie gauche) et une cellule infectée par les herpèsvirus (partie droite). Les cellules non infectées bloquent l'expression des séquences HERV (human endogenous retrovirus), notamment par la méthylation de ces séquences. Les herpèsvirus sont capables de bloquer les méthylases cellulaires, soit par inhibition des enzymes de méthylation (cas de LMP-2A

[latent membrane protein 2] d'EBV), soit par séquestration des méthylases dans le cytoplasme (cas des protéines provenant de HCMV-IE). Les herpèsvirus sont également capables d'induire l'expression des HERV en se fixant sur leurs récepteurs cellulaires, en utilisant notamment les récepteurs des fragments $C 3 b$ et C4b du complément, tels que CD46 ou CD21 (respectivement récepteurs de HHV- 6 et EBV). Dans le cas de HSV-1, certaines protéines virales (ICP) sont capables d'activer les LTR rétroviraux dans l'ADN chromosomique, permettant l'initiation de la transcription de certains HERV. De plus, les herpèsvirus sont indirectement responsables d'une régulation de l'activation de certains HERV via la réponse interféron qu'ils induisent après reconnaissance par les LTR. Cette régulation peut être positive dans le cas de l'IFN- $\alpha$, ou négative dans le cas de I'IFN- $\beta$. IFN : interféron; IFNAR : interferon- $\alpha / \beta$ receptor; TLR : Toll-like receptor; HSV : herpes simplex virus ; HCMV : human cytomegalovirus ; EBV : Epstein-Barr virus; ICP : infected cell protein; LMP : latent membrane protein; IE : immediate early protein; LTR : long terminal repeat.

Barr), HCMV (cytomégalovirus humain), HHV-6 et HHV-8 (Herpesviridae humains 6 et 8 ) ont montré des effets transactivateurs qui s'avèrent d'efficacité variable en fonction des séquences HERV cibles et des tissus ou cellules concernées [2].

L'infection par HSV-l induit, in vitro, la transcription d'éléments HERV-W [2] alors que les virus HHV-6A et -6B transactivent l'expression de l'ARN codant la protéine d'enveloppe HERV-K [4], mais aussi celle d'HERV-W dans certaines cellules (communication personnelle B. Charvet). EBV et HCMV sont eux capables de transactiver respectivement les gènes env et pol des deux éléments HERV-K et HERV-W [5-7]. En effet, la glycoprotéine d'enveloppe d'EBV (la gp350) active la transcription de la famille HERV-W dans les cellules astrocytaires et lymphoïdes [5], montrant ainsi les spécificités multiples, virales, cellulaires et de famille (voire d'éléments) HERV, requises pour que ces transactivations soient effectives.

\section{Rôle de HCMV et EBV sur la méthylation}

Les mécanismes de répression de l'expression des séquences HERV sont variés mais la méthylation des îlots CpG (cytosine-phosphate-guanine) présents dans leur séquence est la mieux décrite. Une levée de cette répression épigénétique par les herpèsvirus pourrait ainsi expliquer la réactivation de certains HERV [8]. Comme représenté dans la Figure 1, HCMV et $\varepsilon B V$ présentent un potentiel «déméthylant» des séquences HERV $[9,10]$. En effet, HCMV peut induire une hypométhylation de la chromatine en rétro-transloquant les $A D N$-méthyltransférases 1 et 3 (DNMT-1, -3) nucléaires dans le cytoplasme permettant I'activation des séquences HERV-K (ou HML-2 [human mouse mammary tumor virus like-2]) [9]. 


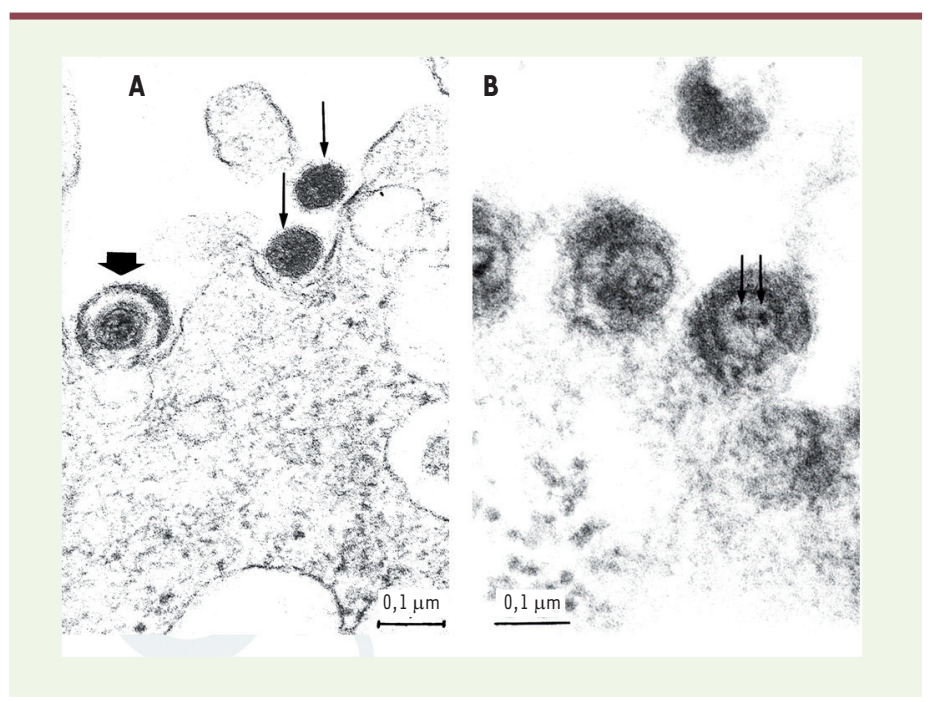

Figure 2. Particules rétrovirales et herpétiques co-exprimées après infection par HSV-1 de cultures de cellules provenant de patients atteints de sclérose en plaques. Des cultures de cellules leptoméningées d'un patient atteint de sclérose en plaques (SEP) ont été infectées par le virus herpès simplex de type 1 (HSV-1). L'observation de ces cellules en microscopie électronique montre, au niveau de la membrane cellulaire: (A) des particules herpétiques typiques - flèche épaisse - ainsi que des particules de type rétroviral - flèches fines ; (B) des particules de la taille de particules herpètiques, mais dont la structure interne ne correspond pas à celle des herpèsvirus. Ces particules présentent un aspect morphologique inhabituel qui pourrait s'expliquer par des interactions lors de la co-expression HERV-herpèsvirus.

\section{Activation des LTR par HSV-1}

HSV-l est connu pour stimuler l'expression de rétrovirus exogènes comme le VIH en activant leur LTR (long terminal repeat) par ses protéines ICP(infected cell polypeptide)0, ICP4, ICP27 et US(unique short)11 [11]. Certaines protéines du virus sont également actives sur la transcription des HERV. En effet, ICPO, en se fixant sur le site AP-1 (activator protein-1) du LTR de HERV-K, et IEl (immediate early protein 1 ), sur le site $0 \mathrm{ct}-1$ (octamer-binding transcription factor) de la région U3 du LTR HERV-W, initient leur transcription [12, 13] $]^{1}$.

Une forte transactivation rétrovirale induite par HSV-l a été observée ex vivo dans des cellules isolées de patients atteints de SEP, après surinfection par le virus ou transfection avec ses gènes «immédiatsprécoces », ICP0 et ICP4 [2, 14]. Cependant, en plus des particules herpétiques et rétrovirales qui sont co-exprimées dans les cultures de cellules leptoméningées ${ }^{2}$ de patients atteints de sclérose en plaques (SEP) après infection par HSV-1, l'observation par microscopie électronique de morphologies anormales suggère la formation de particules hybrides, comme illustré dans la Figure 2. Ceci pose la

${ }^{1}$ Pour une description des rétrovirus, voir [48].

${ }^{2}$ La leptoméninge est la partie des méninges issue des crêtes neurales, et qui comprend la pie-mère et l'arachnoïde. question de l'existence possible d'une interaction entre un herpèsvirus infectieux et un HERV qui pourrait aller au-delà de la simple transactivation, comme pour la vectorisation, déjà décrite, d'un $\varepsilon R V$ animal par le virus de la maladie de Marek $^{3}$, un herpèsvirus aviaire [15].

Les récepteurs des fragments du complément L'engagement des récepteurs membranaires des fragments du complément, notamment le C3b, semble aussi jouer un rôle important dans la transactivation d'HERV. En effet, les gènes de latence d'EBV, LMP (latent membrane protein)-1 et LMP-2A, qui codent des protéines membranaires impliquées dans la survie des lymphocytes $B$ infectés [16], peuvent transactiver HERV-K18 via une interaction avec le CD21 ou CR2 (complement receptor type 2), récepteur du C3d exprimé par les lymphocytes $B$ [17]. Cette transactivation étant quasi immédiate après l'entrée d'દBV dans les cellules, il n'est pas nécessaire qu'il y ait une réplication virale. De même, la transcription du superantigène HERV-K18 serait induite par l'interaction de la glycoprotéine $\mathrm{H}$ de l'herpèsvirus HHV-6B avec le CD46 [18], un récepteur ubiquitaire des fragments $C 3 \mathrm{~b}$ et $\mathrm{C} 4 \mathrm{~b}$ du complément, un dimère immunorégulateur dont la queue cytoplasmique est contituée des sous-unités Cytl ou Cyt2, respectivement anti- et pro-inflammatoire [19]. La modulation de la réponse innée pourrait donc influer sur l'état d'activité des séquences HERV-K.

\section{HERV et physiopathologie : ami ou ennemi ?}

\section{Des protéines endogènes physiologiques ou protectrices}

La mobilité des ERV a participé à l'évolution des espèces et, chez l'homme, des séquences HERV ont été apprivoisées. Ces séquences, bien identifiées, présentent des mutations et des modifications qui leur confèrent une signature moléculaire unique et des propriétés biologiques favorables pour l'organisme hôte.

C'est le cas, maintenant bien étudié, d'une copie HERV-W fixée dans le chromosome 7 humain (HERV-W $7 q$ ) : ses gènes gag et pol sont inactivés, mais le gène env (locus $\varepsilon R V W E-1$ ) présente des mutations et au moins une délétion spécifique par rapport aux autres copies HERV-W env (Figure 3). Ce locus permet l'expression spécifique d'une protéine appelée Syncytine-1 [20] qui est impliquée dans l'implantation embryonnaire et la formation du syncytiotrophoblaste placentaire. Son défaut d'expression est associé à des syndromes de pré-éclampsie [21]. La Syncytine-1

\footnotetext{
${ }^{3}$ Lymphome touchant les gallinacés, en particulier les élevages de poules/poulets.
} 


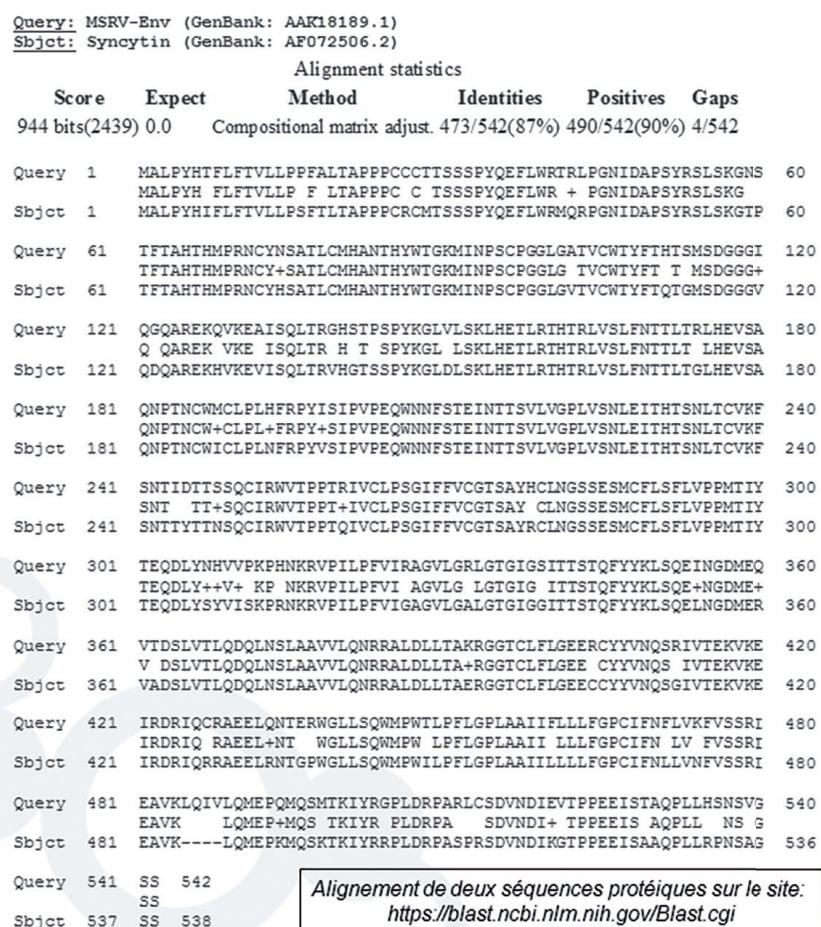

Figure 3. Alignement des séquences d'enveloppes HERV-W provenant de l'ARN de particules virales MSRV (MSRV-env) ou d'ARN placentaire (Syncytine). Les séquences des protéines d'enveloppe HERV-W codées par leurs ARN clonés et séquencés, MSRV (référence GenBank: AAK18189.1; haut) et Syncytine (référence GenBank : AF072506.2 ; bas), ont été alignées avec le logiciel BLAST (https://blast.ncbi.nlm.nih.gov/Blast.cgi). Les acides aminés communs sont présentés entre les deux enveloppes comparées. On note $87 \%$ d'acides aminés identiques et $90 \%$ homologues, avec 4 défauts d'alignement par absence d'acides aminés aux mêmes positions dans la Syncytine, versus MSRV. MSRV : multiple sclerosis-associated retrovirus.

serait aussi régulée par la Suppressyne, une protéine d'enveloppe sans région transmembranaire, ayant pour origine un gène HERV-F intégré dans le chromosome 21 [22]. Bien que les protéines de la famille HERV-W, Syncytine-1 et MSRV-Env (multiple sclerosis retrovirus envelope protein), présentent une homologie de séquence de $90 \%$ (Figure 3 ), elles se localisent différemment après transfection in vitro dans des cellules humaines (Figure 4). Ainsi, au-delà de la régulation physiologique ou pathologique de l'expression de ces protéines HERV-W, quelques différences de séquence suffisent à modifier leurs propriétés biologiques. Un provirus défectif (ne produisant pas de particules virales) comme celui qui code la Syncytine-1 n'en est pas moins actif dans des conditions restreintes, de tissus et de types cellulaires spécifiques. Ces spécificités multiples de facteurs et de conditions, permettant l'activation et l'expression des HERV, sont donc aussi requises pour une expression de copies devenues physiologiques.

L'expression de protéines, voire d'ARN interférents, d'ERV peut s'avérer protectrice contre des copies pathogènes apparentées. Chez la souris, le gène Fv-l (Friend virus-1), relié au gène gag de la famille $\varepsilon R V-L$ murine, a été décrit comme un gène de résistance à l'infection par le virus de la leucémie murine (MLV) [23]. De même, chez le mouton, des copies endogènes du rétrovirus Jaagsiekte (JSRV) sont capables de protéger leur hôte contre la pathogénicité des copies exogènes du même virus, responsable de cancer du poumon à petites cellules (adénomatose ovine pulmonaire) [24]. Des copies «protectrices» analogues pourraient exister chez l'homme, mais cela n'est pas connu à ce jour.

\section{Les protéines endogènes pathogènes}

L'expression de protéines d'HERV est aussi associée à des maladies humaines. Nous prendrons comme exemple la sclérose en plaque (SEP) et la sclérose latérale amyotrophique (SLA) [49] $(\rightarrow)$.

\section{$(\rightarrow)$ Voir la Synthèse de P. Ellul et al., page 404 de ce numéro}

\section{La sclérose en plaque}

L'évaluation histopathologique du tissu cérébral de patient atteint de SEP a montré des lésions inflammatoires démyélinisantes dans la substance blanche du système nerveux central, des défauts de remyélinisation par les cellules précurseurs d'oligodendrocytes (OPC) et des lésions axonales, avec une dégénérescence neuronale dans la substance grise [25]. Ces lésions altèrent la transmission des influx nerveux occasionnant des symptômes variables: pertes de motricité, troubles de la vision, sensibilité altérée ou anormale, troubles de la coordination, etc. Généralement, la SEP évolue par poussées au cours desquelles les symptômes sont exacerbés puis régressent, mais ils finissent par laisser des séquelles. Une progression chronique de la maladie sans récupération fonctionnelle peut alors devenir très invalidante. La SEP touche, selon les régions, entre moins de 30 et plus de 200 personnes sur 100000 avec une proportion de deux femmes pour un homme.

La compréhension globale des facteurs étiologiques qui pourraient directement ou indirectement être impliqués dans la SEP conduit à incriminer une interaction « gèneenvironnement ». Les HERV, qui présentent cette dualité génomique et virale, offrent alors une nouvelle vision de ce que peut être cette interface « gène-environnement ». L'idée qu'un rétrovirus puisse jouer un rôle dans la SEP avait été évoquée mais l'implication d'un rétrovirus exogène classique, donc infectieux, proche du virus humain T-lymphotrope (HTLV-1) avait été suggérée [26]. C'est la raison pour laquelle la découverte d'un nouveau rétrovirus humain dans la SEP (appelé MSRV, multiple sclerosis-associated retrovirus ou élément rétroviral associé à la sclérose en plaques) dont les séquences ont révélé l'existence d'une famille d'HERV 


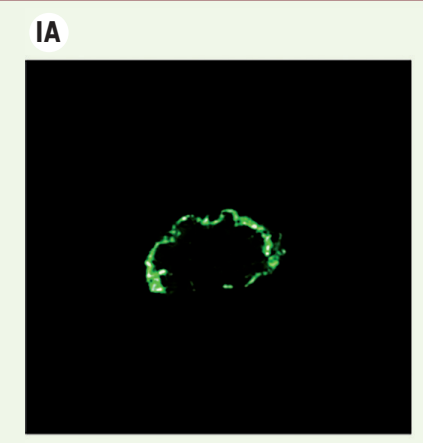

IIA

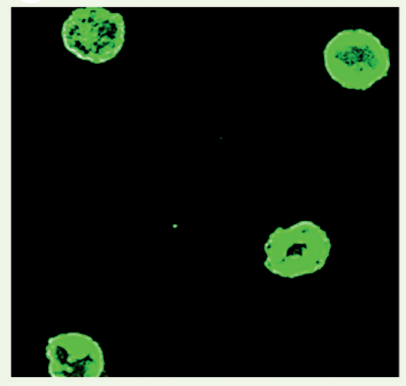

Figure 4. Images de microscopie confocale de cellules HEK exprimant les protéines HERV-W, MSRV-Env ou Syncytine. Les cellules HEK (human embryonic kidney) ont été transfectées par un plasmide (A) codant MSRV-Env ou (B) la Syncytine (HERV7q). (I) Les cellules ont été fixées avec $3 \%$ de paraformaldéhyde et les protéines d'enveloppe ont été détectées avec un anticorps anti-Env fluorescent (GN_mAb-Env03, signal fluorescent vert). (II) Avant la fixation, les cellules ont été perméabilisées par la saponine (0,2\%) pour permettre l'entrée d'anticorps dans les cellules et la détection des protéines d'enveloppe intracellulaires. On observe une détection de MSRV-Env au niveau de la membrane cellulaire, en l'absence de perméabilisation des cellules (I-A), ce qui indique une expression à la surface des cellules. Aucune détection n'est observée avec la Syncytine en l'absence de perméabilisation (I-B). L'expression intracytoplasmique, après perméabilisation, est forte et donne un signal intense péri-membranaire avec MSRV-Env (II-A) mais apparaît faible et reste diffuse pour la Syncytine (II-B). MSRV : multiple sclerosis-associated retrovirus.

jusqu'alors inconnue, puis caractérisée et dénommée HERV-W [27], a initialement été considérée comme anecdotique.

D'autres études ont par la suite exploré et dévoilé progressivement les caractéristiques génétiques et biologiques inattendues de cet HERV. Ainsi, les séquences HERV-W, isolées de particules rétrovirales provenant de cultures de cellules de patients atteints de SEP, codent une protéine d'enveloppe biologiquement active (MSRV-Env) qui est capable d'activer les cascades physiopathologiques à l'origine des signes pathognomoniques de la SEP. En particulier, MSRV-Env peut induire une activation de type superantigène (activation polyclonale, donc disproportionnée, indépendante de la spécificité de l'antigène reconnu) des lymphocytes T [28], elle-même conditionnée en amont à une stimulation de l'immunité innée impliquant le récepteur TLR4 (Tolllike receptor 4) avec des effets pro-inflammatoires [29]. MSRV-Env peut promouvoir l'activation des macrophages, la différenciation des cellules dendritiques, une réponse auto-immune anti-myéline et peut induire une encéphalomyélite auto-immune expérimentale dans le modèle animal expérimental de la SEP [30]. MSRV-Env agit également sur les cellules endothéliales vasculaires cérébrales, qui expriment TLR4, affectant la perméabilité vasculaire et l'expression de récepteurs d'adhésion cellulaire (ICAM-1, intercellular adhesion molecule 1), ce qui contribue à l'invasion du système nerveux par les leucocytes circulants [31].

Sur les cellules gliales, les particules MSRV induisent un effet cytopathique avec une activité transcriptase inverse (RT) [32]. Les mécanismes moléculaires correspondants n'ont pu être élucidés jusqu'à la démonstration récente que l'interaction entre MSRVEnv et TLR4, exprimé transitoirement dans les OPC, provoque un blocage de leur différenciation et, en conséquence, l'arrêt du processus de remyélinisation [33]. La détection systématique de MSRV-Env associée aux cellules microgliales et macrophagiques dans les zones de démyélinisation cérébrales actives de SEP, désignent cette protéine comme une cible thérapeutique potentielle [34].

Une nouvelle approche thérapeutique est actuellement évaluée avec un anticorps monoclonal humanisé neutralisant l'activité pathogène de MSRV-Env, in vitro et in vivo, au niveau immuno-inflammatoire [35], mais aussi en ce qui concerne le blocage de la remyélinisation par les OPC [36]. Cet anticorps, le $\mathrm{GNbACl}$, est actuellement en phase Ilb des essais cliniques (étude réglementaire d'efficacité) chez des patients atteints de SEP. Ceci fait suite aux études de phase I et lla pour évaluer la sécurité et la tolérance médicamenteuse ainsi que la pharmacocinétique du produit chez des volontaires sains, puis chez des patients atteints de SEP, chez lesquels une injection mensuelle de GNbACl pendant un an n'a montré aucune perturbation de leurs fonctions immunitaires [37]. D'autres approches thérapeutiques fondées sur des antirétroviraux utilisés pour le traitement de rétrovirus exogènes comme le VIH, n'ont pas donné de résultat positif à ce jour.

\section{La sclérose latérale amyotrophique}

La SLA (encore appelée maladie de Charcot) est une maladie dégénérative du neurone moteur du cortex, du tronc cérébral et de la moelle épinière. La SLA provoque une paralysie progressive de la majorité de la musculature squelettique impliquée dans la motricité volontaire. L'aggravation de la maladie est actuellement irréversible et le décès, souvent par insuffisance respiratoire, survient pour plus de $70 \%$ des patients dans les 3 à 5 ans après le diagnostic [38]. 
La SLA est actuellement classée comme une maladie des protéines, ou «protéinopathie », au même titre que d'autres maladies neurodégénératives (prion, Alzheimer, Parkinson, démence à corps de Lewy, maladie de Huntington) [39]. Dans plus de $90 \%$ des cas, la SLA est en effet associée à la présence d'inclusions cytoplasmiques majoritairement composées d'une forme pathologique de la protéine cellulaire Tar DNA binding protein 43 (TDP-43) mal conformée dans les neurones moteurs et les oligodendrocytes [40]. L'origine de la SLA est certainement complexe. On estime que $90 \%$ des cas sont sporadiques et $10 \%$ sont héréditaires. Ces derniers sont associés à des mutations dans des gènes comme c9orf72 (chromosome 9 open reading frame 72) ou tdp43 [41] alors que les formes sporadiques restent encore à ce jour très peu comprises.

Dès les années 1970, l'hypothèse d'une infection virale à l'origine de la SLA a été suspectée. Puis les rétrovirus ont été privilégiés en raison de la découverte d'une activité transcriptase inverse (RT) d'origine rétrovirale dans le cerveau et dans le sang de patients atteints de SLA [42].

Des études menées sur des cohortes de patients atteints de SLA sporadique mais séronégatifs pour les rétrovirus infectieux connus, ont indiqué que plus de $50 \%$ d'entre eux présentaient dans leur sang une activité RT aussi importante que celle mesurée chez des individus infectés par le VIH-l, contre seulement $10 \%$ chez des individus contrôles [43]. C'est en 2011 que l'équipe d'Avindra Nath a identifié et caractérisé la famille de rétrovirus endogènes humains HERV-K comme étant l'origine de cette activité RT [44]. Au cours de ces études, une activation spécifique de HERV-K a été mise en évidence dans les neurones des patients atteints de SLA corrélée à une augmentation des ARN viraux (gag-pol et env). Les glycoprotéines d'enveloppe ( $\varepsilon n v)$ et la RT ont également été détectées. Dans des cultures de neurones humains, et chez des souris transgéniques, la glycoprotéine d'enveloppe HERV-K induit une dégénérescence des neurones moteurs associée à une diminution du cortex moteur, de l'activité synaptique et à une atrophie musculaire conduisant à un déficit moteur important [45]. Par ailleurs, d'autres études ont indiqué qu'in vitro, l'expression de HERV-K était régulée par la protéine TDP-43 [46] et récemment, Manghera et al. ont observé que TDP-43, sous sa forme agrégée pathologique, pouvait induire l'accumulation de protéines virales HERV-K dans les neurones [47]. L'ensemble de ces données expérimentales suggèrent donc une relation étroite entre, d'une part, la protéine TDP-43 et ses formes mutantes et le rétrovirus endogène $H \varepsilon R V-K$, d'autre part, dans la pathogénie de la SLA.

L'activation de HERV-K semble donc participer au processus pathologique de la SLA. Cependant, comme avec HERV-W pour la SEP, il est concevable que des facteurs multiples et variables de l'environnement puissent être des « initiateurs » d'une activation ciblée, puis auto-entretenue, de copies HERV-K dont la glycoprotéine d'enveloppe serait alors motrice dans le processus pathologique de la SLA. Les études en cours apporteront des réponses qui devraient permettre de préciser ou de modifier cette hypothèse.

\section{Conclusions et perspectives}

On comprend aujourd'hui que les séquences provenant des éléments génétiques mobiles, qui furent probablement un moteur moléculaire de l'évolution des génomes et le restent peut-être encore, comme cela a été montré chez le koala [48] $(\rightarrow)$ ont laissé des vestiges considérables dans le génome humain. En effet, elles représentent environ la moitié $(\rightarrow)$ Voir la Synthèse de J. Medina et $H$. Perron, $m / s n^{\circ} 2$, février 2017, page 151 des séquences $A D N$ génomiques humaines et, bien que l'immense majorité soit devenue inactive par l'accumulation de mutations et de modifications somatiques de leur ADN, certaines ont conservé des capacités fonctionnelles partielles ou complètes.

Ces «vestiges » sont majoritaires dans notre génome car les séquences codant les protéines physiologiques ou somatiques représentent moins de $3 \%$ du génome humain. Les plus complexes proviennent d'insertions germinales d'origine rétrovirale (HERV) qui représentent environ $8 \%$ des séquences de I'ADN humain. Les HERV font actuellement l'objet d'un intérêt particulier depuis la mise en évidence de leur implication dans des fonctions tant physiologiques que pathologiques. Les premières se révèlent essentielles à l'homme puisque dans le processus de reproduction, la protéine d'enveloppe HERV-W «domestiquée » (la Syncytine-1) s'avère critique pour la placentation et, au-delà, pour le développement fœtal. Les secondes permettent d'identifier de nouvelles molécules impliquées dans la pathogénie de maladies aujourd'hui incurables dont elles peuvent être un dénominateur commun en amont des cascades physiopathogéniques initiées par des facteurs environnementaux. Ainsi, de nouvelles cibles thérapeutiques, avec des molécules inhibitrices ou neutralisantes des interactions spécifiques avec des récepteurs impliqués dans la pathogénie, sont maintenant identifiées et étudiées dans des maladies comme la SEP et la SLA. Ceci offre déjà des perspectives encore inconcevables avant les découvertes de ces dernières décennies dans le domaine des HERV.

En conclusion, et quoiqu'il en soit des études cliniques actuelles, le domaine scientifique défini par les séquences génétiques mobiles représente un nouveau champ d'exploration extrêmement vaste et fort méconnu en biologie humaine. Cette terra incognita est probablement une nouvelle et immense frontière pour la recherche et l'innovation thérapeutique des temps à venir. $\diamond$

\section{SUMMARY}

Endogenous retroviral sequences in the human genome can play a physiological or pathological role Human endogenous retroviruses (HERV) represent a large part of our genome and the few elements that have retained a potential of expression still remain "dormant" in physiological conditions. In some instances, they can be awakened by environmental factors activating their expression. The best studied conditions of HERV activation are infections caused by microorganisms such as 
viruses of the Herpesvirus family. This activation can thus lead to the expression of pathogenic proteins such as envelope proteins belonging to the HERV-W and HERV-K families, respectively involved in Multiple Sclerosis (MS) and amyotrophic lateral sclerosis (ALS). Endogenous retroviral proteins can also acquire a physiological function beneficial for humans. This is the case of Syncytin-1 from the HERV-W family, that is involved in placenta formation. $\diamond$

\section{LIENS D'INTÉRÊT}

J. Medina et H. Perron déclarent avoir des liens durables avec l'entreprise GeNeuroInnovation, 60, avenue Rockefeller, 69008 Lyon, France.

B. Charvet, P. Leblanc, R. Germi, B. Horvat et P.N. Marche déclarent n'avoir aucun lien d'intérêt concernant les données publiées dans cet article.

\section{RÉFÉRENCES}

1. Leboyer M, Tamouza R, Charron D, et al. Human endogenous retrovirus type W (HERV-W) in schizophrenia: a new avenue of research at the gene-environment interface. World J Biol Psychiatry 2013; $14: 80-90$.

2. Perron $H$, Lang $A$. The human endogenous retrovirus link between genes and environment in multiple sclerosis and in multifactorial diseases associating neuroinflammation. Clin Rev Allergy Immunol $2010 ; 39: 51-61$.

3. Engel ME, Hiebert SW. The enemy within: dormant retroviruses awaken. Nat Med $2010 ; 16: 517-8$.

4. Reynaud J, M P, Horvat B, et al. Human Herpesvirus 6 and neuroinflammation. ISRN Virology 2013; 2013 : e834890.

5. Mameli G, Poddighe L, Mei A, et al. Expression and activation by Epstein Barr virus of human endogenous retroviruses $-W$ in blood cells and astrocytes: inference for multiple sclerosis. PLoS One $2012 ; 7$ : e44991.

6. Sutkowski N, Conrad B, Thorley-Lawson DA, et al. Epstein-Barr virus transactivates the human endogenous retrovirus HERV-K18 that encodes a superantigen. Immunity $2001 ; 15: 579-89$.

7. Bergallo M, Galliano I, Montanari $P$, et al. CMV induces HERV-K and HERV-W expression in kidney transplant recipients. J Clin Virol $2015 ; 68: 28-31$.

8. Lavie L, Kitova M, Maldener $\varepsilon$, et al. CpG methylation directly regulates transcriptional activity of the human endogenous retrovirus family HERV-K(HML-2).J Virol $2005 ; 79: 876-83$.

9. Esteki-Zadeh A, Karimi M, Strååt $K$, et al. Human cytomegalovirus infection is sensitive to the host cell DNA methylation state and alters global DNA methylation capacity. Epigenetics $2012 ; 7: 585-93$.

10. Niller HH, Tarnai Z, Decsi G, et al. Role of epigenetics in $\varepsilon B V$ regulation and pathogenesis. Future Microbiol $2014 ; 9: 747-56$.

11. Palù G, Benetti L, Calistri A. Molecular basis of the interactions between herpes simplex viruses and HIV-1. Herpes J IHMF $2001 ; 8: 50-5$.

12. Kwun HJ, Han HJ, Lee WJ, et al. Transactivation of the human endogenous retrovirus $\mathrm{K}$ long terminal repeat by herpes simplex virus type 1 immediate early protein 0 . Virus Res $2002 ; 86: 93-100$.

13. Lee WJ, Kwun HJ, Kim HS, et al. Activation of the human endogenous retrovirus W long terminal repeat by herpes simplex virus type 1 immediate early protein 1 . Mol Cell $2003 ; 15: 75-80$.

14. Perron $H$, Suh M, Lalande B, et al. Herpes simplex virus ICPO and ICP4 immediate early proteins strongly enhance expression of a retrovirus harboured by a leptomeningeal cell line from a patient with multiple sclerosis. J Gen Virol 1993 ; $74: 65-72$.

15. Isfort $R$, Jones $D$, Kost $R$, et al. Retrovirus insertion into herpesvirus in vitro and in vivo. Proc Natl Acad Sci USA $1992 ; 89$ : 991-5.

16. Caldwell RG, Wilson JB, Anderson SJ, et al. Epstein-Barr virus LMP2A drives B cell development and survival in the absence of normal B cell receptor signals. Immunity $1998 ; 9: 405-11$.

17. Sutkowski N, Chen G, Calderon G, et al. Epstein-Barr virus latent membrane protein LMP-2A is sufficient for transactivation of the human endogenous retrovirus HERV-K18 superantigen. J Virol $2004 ; 78: 7852-60$.

18. Turcanova VL, Bundgaard B, Höllsberg P. Human herpesvirus-6B induces expression of the human endogenous retrovirus K18-encoded superantigen. J Clin Virol 2009 ; $46: 15-9$.

19. Marie JC, Astier AL, Rivailler P, et al. Linking innate and acquired immunity: divergent role of CD46 cytoplasmic domains in T cell induced inflammation. Nat Immunol $2002 ; 3: 659-66$.

20. Mi S, Lee $X$, Li X, et al. Syncytin is a captive retroviral envelope protein involved in human placental morphogenesis. Nature 2000 ; $403: 785-9$.

21. Lee X, Keith JC, Stumm N, et al. Downregulation of placental syncytin expression and abnormal protein localization in pre-eclampsia. Placenta $2001 ; 22: 808-12$.

22. Sugimoto J, Sugimoto $M$, Bernstein $\mathrm{H}$, et al. A novel human endogenous retroviral protein inhibits cell-cell fusion. Sci Rep $2013 ; 3: 1462$.

23. Yan Y, Buckler-White A, Wollenberg K, et al. Origin, antiviral function and evidence for positive selection of the gammaretrovirus restriction gene $\mathrm{Fvl}$ in the genus Mus. Proc Natl Acad Sci USA $2009 ; 106: 3259-63$.

24. Varela M, Spencer TE, Palmarini M, et al. Friendly viruses: the special relationship between endogenous retroviruses and their host. Ann NY Acad Sci $2009 ; 1178: 15772$.
25. Chang A, Tourtellotte WW, Rudick R, et al. Premyelinating oligodendrocytes in chronic lesions of multiple sclerosis. N Engl J Med $2002 ; 346: 16573$.

26. Koprowski H, DeFreitas $E C$, Harper ME, et al. Multiple sclerosis and human T-cell lymphotropic retroviruses. Nature $1985 ; 318$ : 154-60.

27. Blond JL, Besème F, Duret $L$, et al. Molecular characterization and placental expression of HERV-W, a new human endogenous retrovirus family. J Virol $1999 ; 73: 1175-85$.

28. Perron $\mathrm{H}$, Jouvin-Marche $\varepsilon$, Michel M, et al. Multiple sclerosis retrovirus particles and recombinant envelope trigger an abnormal immune response in vitro, by inducing polyclonal Vbetal6 T-lymphocyte activation. Virology $2001 ; 287: 321-32$.

29. Rolland A, Jouvin-Marche $\varepsilon$, Viret C, et al. The envelope protein of a human endogenous retrovirus- $W$ family activates innate immunity through CD14/ TLR4 and promotes Thl-like responses. J Immunol 2006 ; 176 : 7636-44.

30. Perron H, Dougier-Reynaud H-L, Lomparski C, et al. Human endogenous retrovirus protein activates innate immunity and promotes experimental allergic encephalomyelitis in mice. PLoS One 2013; 8 : e80128.

31. Duperray A, Barbe D, Raguenez G, et al. Inflammatory response of endothelial cells to a human endogenous retrovirus associated with multiple sclerosis is mediated by TLR4. Int Immunol $2015 ; 27$ : 545-53.

32. Ménard A, Amouri R, Michel M, et al. Gliotoxicity, reverse transcriptase activity and retroviral RNA in monocyte/macrophage culture supernatants from patients with multiple sclerosis. FEBS Lett 1997 ; 413 : 477-85.

33. Kremer D, Schichel T, Förster M, et al. Human endogenous retrovirus type $W$ envelope protein inhibits oligodendroglial precursor cell differentiation. Ann Neurol $2013 ; 74: 721-32$.

34. Horssen J van, Pol S van der, Nijland P, et al. Human endogenous retrovirus $W$ in brain lesions: Rationale for targeted therapy in multiple sclerosis. Mult Scler Relat Disord $2016 ; 8: 11-8$.

35. Curtin F, Perron H, Kromminga A, et al. Preclinical and early clinical development of $\mathrm{GNbACl}$, a humanized IgG4 monoclonal antibody targeting endogenous retroviral MSRV-Env protein. mAbs $2015 ; 7: 265-75$.

36. Kremer $D$, Förster $M$, Schichel $T$, et al. The neutralizing antibody GNbACl abrogates HERV-W envelope protein-mediated oligodendroglial maturation blockade. Mult Scler Houndmills Basingstoke Engl 2015 ; 21 : 1200-3.

37. Zimmermann M, Sanderson NSR, Rasenack M, et al. Immunologic monitoring during a phase $2 a$ trial of the GNbACl antibody in patients with MS. Neuro Neuroimmunol Neuroinflammation 2015 ; 2 : el44.

38. Hardiman 0, Berg LH van den, Kiernan MC. Clinical diagnosis and management of amyotrophic lateral sclerosis. Nat Rev Neurol $2011 ; 7: 639-49$.

39. Polymenidou M, Cleveland DW. The seeds of neurodegeneration: prion-like spreading in ALS. Cell $2011 ; 147:$ 498-508.

40. Neumann M, Sampathu DM, Kwong LK, et al. Ubiquitinated TDP-43 in frontotemporal lobar degeneration and amyotrophic lateral sclerosis. Science $2006 ; 314: 130-3$.

41. Turner MR, Hardiman 0 , Benatar M, et al. Controversies and priorities in amyotrophic lateral sclerosis. Lancet Neurol $2013 ; 12$ : 310-22.

42. Viola MV, Frazier M, White L, et al. RNA-instructed DNA polymerase activity in a cytoplasmic particulate fraction in brains from Guamanian patients. J Exp Med $1975 ; 142:$ 483-94.

43. McCormick AL, Brown RH, Cudkowicz ME, et al. Quantification of reverse transcriptase in ALS and elimination of a novel retroviral candidate. Neurology $2008 ; 70: 278-83$.

44. Douville R, Liu J, Rothstein J, et al. Identification of active loci of a human endogenous retrovirus in neurons of patients with amyotrophic lateral sclerosis. Ann Neurol $2011 ; 69$ : 141-51.

45. Li W, Lee MH, Henderson L, et al. Human endogenous retrovirus- $K$ contributes to motor neuron disease. Sci Transl Med $2015 ; 7$ : 307 ral53.

46. Saldi TK, Ash PE, Wilson G, et al. TDP-1, the Caenorhabditis elegans ortholog of TDP-43, limits the accumulation of double-stranded RNA. EMBO J 2014 ; $33: 2947-66$.

47. Manghera M, Ferguson-Parry J, Douville RN. TDP-43 regulates endogenous retrovirus-K viral protein accumulation. Neurobiol Dis 2016; 94 : 226-36.

48. Medina J, Perron H. Séquences provenant d'éléments génétiques mobiles, face cachée du génome humain. Med Sci (Paris) 2017 ; 33 : 151-8.

49. Ellul $P$, Groc L, Leboyer $M$. Les rétrovirus endogènes humains, une implication dans la schizophrénie et le trouble bipolaire. Med Sci (Paris) 2017 ; 33 : 404-9.
TIRÉS À PART

H. Perron 